\title{
Contraceptive nonuse among women in Uganda: a comparative assessment of predictors across regions
}

\author{
Jude Otim* (1)
}

\begin{abstract}
Background: Contraceptive nonuse has diverse effects on women, such as unintended pregnancies and births that result in high fertility and poor maternal health outcomes. In Uganda, knowledge on contraceptive use is high, amidst undesirably high contraceptive nonuse and scarce literature on predictors of contraceptive nonuse across regions. This study assessed factors associated with contraceptive nonuse among women of reproductive age across regions in Uganda.

Method: This study used data from a cross-sectional 2016 Uganda demographic and health survey that had 18,506 women of reproductive age. The relationship between contraceptive nonuse and socio-economic and demographic factors across regions were assessed using a binary multivariable logistic regression model.

Results: In Uganda, contraceptive nonuse is estimated at 40\%. Northern region (55\%) had the highest prevalence of contraceptive nonuse compared to Central region (35\%) with the lowest. Across regions, wealth index, number of living children, educational level, and children born in the last 5 years prior to the demographic survey differently predicted contraceptive nonuse. Conversely, age, religion, age at first marriage, sexual autonomy, age at first birth, desire for children, listening to radio, and employment status were only predictors of contraceptive nonuse in particular regions amidst variations. Residence, perception of distance to health facility, watching television, and reading newspapers or magazines did not predict contraceptive nonuse.
\end{abstract}

Conclusions: The study findings propose the need to appreciate regional-variations in effect of contraceptive nonuse predictors and therefore, efforts should be directed towards addressing regional-variations so as to attain high contraceptive usage across regions, and thus reduce on unwanted pregnancies and births.

Keywords: Contraceptive nonuse, Regional, Fertility, Women, Uganda

\section{Background}

Worldwide, an estimated 190 million women (1549 years) do not use contraceptives; most of whom are found in sub-Saharan Africa (83\%) [1, 2]. Contraceptive nonuse among women of reproductive age in sub-Saharan Africa accounts for nearly 14

*Correspondence: otimjude18@gmail.com Department of Sociology and Social Administration, Kyambogo

University, P.O. Box 1, Kyambogo, Kampala, Uganda million unplanned pregnancies annually and majority of maternal deaths (66\%) [3-7], amidst geographical variations $[8,9]$. Uganda continues to present undesirable fertility rates (5.4 births per woman) and maternal mortality ratios (336 maternal deaths per 100,000 live births) $[10,11]$, that are associated with contraceptive nonuse [12-17]. In Uganda, almost everybody (99\%) has knowledge on contraceptive use [10]. However, the knowledge is not equitable to current contraceptive 
uptake (39\%) [10]; attributed to indistinct factors, particularly across regions of the country.

Studies on contraceptive nonuse in Uganda report socio-economic and demographic preditors such as; educational level, age, wealth status, fear of side effects, residence, low quality of contraceptive services, alcohol intake, income, sex, and age at first sex [16, 18-20], without examining predictors' across regions. Conspicuously, wide variations in contraceptive nonuse, and consequent fertility, and maternal mortality exist within regions in Uganda despite continued good strategies and rigorous efforts to lower contraceptive nonuse in the country [10, 21]. For instance; Karamoja region has the highest prevalence of contraceptive nonuse (92.7\%) among currently married women (15-49 years), whilst highest fertility (7.9 children per woman) and maternal mortality ratio (588 per 100,000 live births), compared to other regions [10]. Therefore, differences in contraceptive nonuse across regions suggest regional-specific predictors of the vice.

Uganda is composed of four administrative regions, and they include; Eastern, Northern, Central, and Western regions [22]. These regions have variations in livelihood sources, as well as levels of poverty [22]. For instance; Eastern region is known to be the poorest of all regions in the country [23]. In addition, Karamoja region is reportedly the least social and economically developed region [24]. Studies indicate that discrepancies in poverty and sources of livelihood have a bearing on contraceptive nonuse $[23,25,26]$. Therefore, this study underscores the need to seek address of this gap through examining the association between contraceptive nonuse among women (15-49 years) and socioeconomic and demographic factors across regions of the country.

\section{Methods}

\section{Data used}

This paper used secondary data from the 2016 Uganda Demographic and Health Survey (UDHS). Details regarding sampling in the 2016 UDHS can be obtained elsewhere [10]. In the present study, I obtained access and permission to download and use the 2016 UDHS data from DHS program web platform, after submitting the study proposal. This study utilized the women's questionnaire that focused on women of reproductive age (15-49 years). In the 2016 UDHS women's questionnaire, women of reproductive age were asked about whether they have ever used anything or tried to delay or avoid getting pregnant; this was used as a measure of contraceptive nonuse in this study. This study incorporated all women aged 15-49 $(18,506)$; these women are exposed to the risk of pregnancy [10]. Women utilize contraceptives in order to reduce on the risk of unwanted pregnancies and child birth [27-29]. This study regrouped the fifteen (15) regions in the 2016 UDHS into four (4) regions of Uganda for the rationale of analysis; Central (Kampala, south Buganda, and North Buganda), Eastern (Busoga, Bukedi, Bugisu, and Teso), Western (Bunyoro, Tooro, Ankole, and Kigezi), and Northern regions (Lango, Acholi, Karamoja, and West nile) [28, 30]. In order to ensure that the sampled data was representative, and adjusted for non-responses in the country, data was weighted and compound design in analyses while utilizing the SVY command in STATA 13.0 was considered. Figure 1 is a flow diagram that indicates the derivation of the study sample.

\section{Variables}

The dependent variable of this study was contraceptive nonuse. This was assessed using a binary outcome; whether a woman has never used anything or tried to delay or avoid getting pregnant (coded 1 ), and or has ever

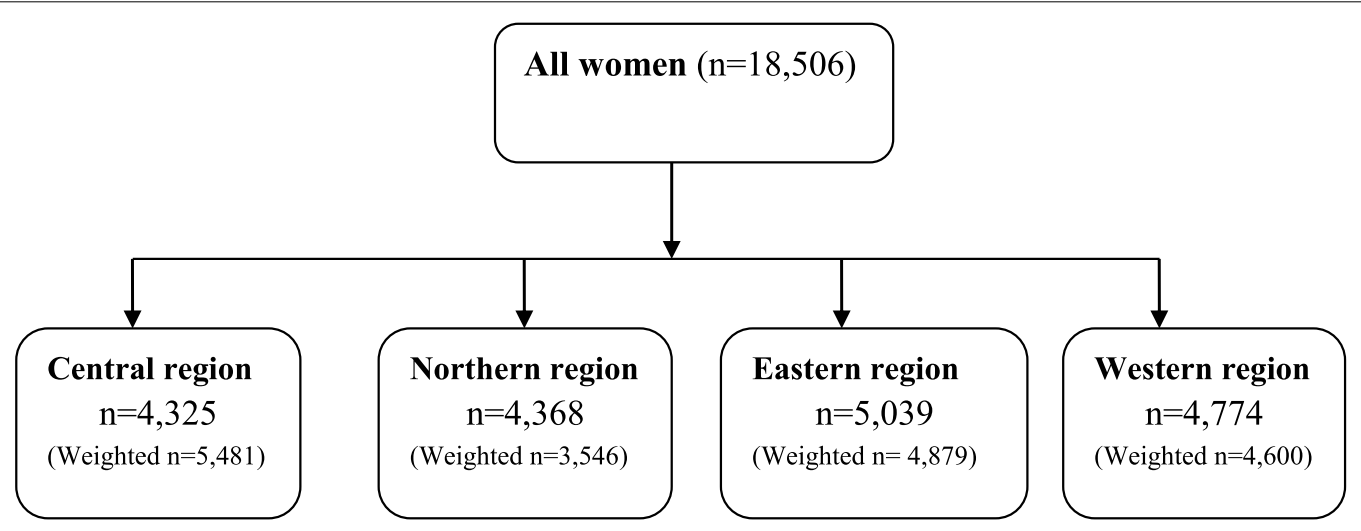

Fig. 1 Origin of the sample adopted for the study 
used anything or tried to delay or avoid getting pregnant (coded 0). In this study, the independent variables used include; age of the respondent, respondent's desire for children, respondent's number of living children, number of children born to the respondent in the last 5 years, employment status of the respondent, respondent's education level, respondent's place of residence, religious affiliation of the respondents, household wealth index, age at first marriage, age at first sex, radio listening, television watching, reading newspaper or magazines, perception of distance to health facility, sexual autonomy and age at first birth. Women who reported a frequency of having sexual freedom and, listening/watching and reading newspapers or magazines were grouped as "yes" whereas women who reported no frequency were grouped as "no".

\section{Statistical analysis}

Analysis was done using STATA 13.0. Three stages were undertaken, which included; first, with the use of frequency distributions, we created descriptive summaries on women's demographic and socio-economic characteristics across all the regions of Uganda. Second, analysis of variation in contraceptive nonuse by women's demographic and socio-economic factors across all the regions of the country was done through a cross-tabular analysis with relationships investigated using Pearson Chi-square test. Third, net-association of women's demographic and socio-economic characteristics on contraceptive nonuse was done with a logistic regression analysis to obtain the likelihood estimates of contraceptive nonuse among women across all the regions of Uganda. Logistic regression was adopted due to the nature of the modelled outcome variable (binary outcome). Odds ratios (OR's) with 95\% confidence interval were adopted in the presentation of study findings. Relationships with $p$ values $<0.05$ were considered statistical significant; additionally, $p$ value $<0.001$ indicated very strong relationships, $p$ value $<0.01$ showed a strong relationship, and $p$ value $<0.05$ indicated moderate relationships [31]. Archer-Lemeshow goodness of fit test was adopted in testing the suitability of the regression model using STATA 13.0 software [32].

\section{Results}

\section{Descriptive findings of the respondents}

Results (Table 1) indicate statistical significance of contraceptive nonuse across regions of the country. In the results, reveal the highest prevalence of contraceptive nonuse was in Northern region (55\%), followed-by Western region (44\%), and lowest in Central region (35\%).
Table 1 Percentage distribution of contraceptives nonuse among women aged 15-49 years across regions

\begin{tabular}{lllll}
\hline Fertility & Regions & & & \\
\cline { 2 - 5 } & $\begin{array}{l}\text { Central } \\
(\mathbf{n}=\mathbf{4 3 2 5})\end{array}$ & $\begin{array}{l}\text { Eastern } \\
(\mathbf{n}=\mathbf{5 0 3 9})\end{array}$ & $\begin{array}{l}\text { Northern } \\
(\mathbf{n}=\mathbf{4 3 6 8})\end{array}$ & $\begin{array}{l}\text { Western } \\
(\mathbf{n}=\mathbf{4 7 7 4})\end{array}$ \\
\hline $\begin{array}{l}\text { Contraceptive } \\
\text { nonuse }\end{array}$ & $1491(35 \%)$ & $2153(43 \%)$ & $2420(55 \%)$ & $2084(44 \%)$ \\
\begin{tabular}{l} 
Contraceptive use \\
\hline
\end{tabular} & $2834(65 \%)$ & $2886(57 \%)$ & $1947(45 \%)$ & $2690(56 \%)$ \\
\hline
\end{tabular}

$x^{2}=386.6, p=0.0000$

Table 1 shows a distribution of contraceptive nonuse among women (15-49 years) across regions.

Results in Table 2 indicate selected women's socioeconomic and demographic characteristics across regions. Most respondents have primary educational level, with the highest proportion in Northern region (67.1\%). Also, Northern region (49.8\%) unlike other regions had the highest proportion of women in the poorest wealth index. Across regions, more than half of the respondents reside in the rural areas. Additionally, the results also reveal that several respondents across regions married before their 18 birthday; with majority of respondents in Northern region (51\%), followedby Eastern region $(48 \%)$, and lowest in Central region (37.9\%). In addition, results depict that over $50 \%$ of the respondents across all regions perceived no problem with distance to health facility. Besides, majority of the respondents were unemployed, with highest proportion in Northern region (80.7\%).

\section{Analysis of variations in respondent's socio-economic and demographic predictors by contraceptive nonuse across regions}

This study examined socio-economic and demographic predictors of contraceptive nonuse at bivariate level of analysis. At this level, associations were investigated using Pearson chi-square test that compared differences in contraceptive nonuse by socio-economic and demographic predictors across regions. In the results (Table 3), respondents' age, age at first sex, number of children born in the last five (5) years, listening to radio and employment status was statistically significant across regions $(p<0.05)$. Educational level, religious status, age at first marriage, desire for children, sexual autonomy, wealth quintile, residence, age at first birth, and watching television were only statistically significant in some regions. For perception of distance to the health facility and reading newspapers or magazines, these were not significant across regions. Therefore, due to the importance of all the variables in predicting 
Table 2 Percentage distribution of women's socio-economic and demographic factors across regions

\begin{tabular}{|c|c|c|c|c|}
\hline \multirow[t]{2}{*}{ Characteristics } & \multicolumn{4}{|l|}{ Regions } \\
\hline & $\begin{array}{l}\text { Central } \\
(n=4325)\end{array}$ & $\begin{array}{l}\text { Eastern } \\
(n=5039)\end{array}$ & $\begin{array}{l}\text { Northern } \\
(n=4368)\end{array}$ & $\begin{array}{l}\text { Western } \\
(n=4600)\end{array}$ \\
\hline \multicolumn{5}{|l|}{ Age } \\
\hline $15-19$ & 894.4 (20.7\%) & 1288.7 (25.6\%) & $1111.1(25.4 \%)$ & $1019.3(21.4 \%)$ \\
\hline $20-24$ & $993.9(23.0 \%)$ & $1014.4(20.1 \%)$ & $834.9(19.1 \%)$ & 936.5 (19.6\%) \\
\hline $25-29$ & 791.0 (18.3\%) & 741.3 (14.7\%) & $669.4(15.3 \%)$ & $817.7(17.1 \%)$ \\
\hline $30-34$ & $572.6(13.2 \%)$ & $652.0(12.9 \%)$ & $633.9(14.5 \%)$ & $697.0(14.6 \%)$ \\
\hline $35-39$ & $485.9(11.2 \%)$ & $505.5(10.0 \%)$ & $455.0(10.4 \%)$ & $556.9(11.7 \%)$ \\
\hline $40-44$ & $338.8(7.8 \%)$ & 489.8 (9.7\%) & $385.5(8.8 \%)$ & $406.0(8.5 \%)$ \\
\hline $45-49$ & $249.5(5.8 \%)$ & 347.5 (6.9\%) & $278.2(6.4 \%)$ & 340.7 (7.1\%) \\
\hline Total (\%) & 100 & 100 & 100 & 100 \\
\hline \multicolumn{5}{|l|}{ Educational level } \\
\hline No education & $231.3(5.4 \%)$ & $352.1(7.0 \%)$ & $751.1(17.2 \%)$ & $557.8(11.7 \%)$ \\
\hline Primary & $1862.4(43.1 \%)$ & $3116.0(61.8 \%)$ & $2931.4(67.1 \%)$ & 2981.7 (62.5\%) \\
\hline Secondary & $1613.3(37.3 \%)$ & $1293.2(25.7 \%)$ & $495.2(11.3 \%)$ & $975.9(20.4 \%)$ \\
\hline Higher & $618.0(14.3 \%)$ & $277.7(5.5 \%)$ & $190.3(4.4 \%)$ & $258.7(5.4 \%)$ \\
\hline Total (\%) & 100 & 100 & 100 & 100 \\
\hline \multicolumn{5}{|l|}{ Religion } \\
\hline Catholic & 1511.0 (34.9\%) & $1403.9(27.9 \%)$ & $2587.1(59.2 \%)$ & $2034.7(42.6 \%)$ \\
\hline Anglican & 1138.6 (26.3\%) & $1845.6(36.6 \%)$ & $991.0(22.7 \%)$ & $1805.5(37.8 \%)$ \\
\hline Muslim & 794.2 (18.4\%) & $866.1(17.2 \%)$ & $409.8(9.4 \%)$ & $218.6(4.6 \%)$ \\
\hline Pentecostal & 742.5 (17.2\%) & 799.1 (15.9\%) & 360.6 (8.3\% & 478.1 (10.0\%) \\
\hline Others & $138.8(3.2 \%)$ & $124.3(2.5 \%)$ & $19.6(0.5 \%)$ & $237.1(5.0 \%)$ \\
\hline Total (\%) & 100 & 100 & 100 & 100 \\
\hline \multicolumn{5}{|c|}{ Age at first marriage } \\
\hline$\leq 12$ & $90.4(3.0 \%)$ & $140.5(3.7 \%)$ & $119.6(3.6 \%)$ & $142.0(3.9 \%)$ \\
\hline $13-17$ & 1150.9 (37.9\%) & $1811.1(48.0 \%)$ & $1706.8(51.0 \%)$ & $1474.8(40.8 \%)$ \\
\hline $18-24$ & 1519.4 (50.1\%) & $1578.5(41.8 \%)$ & $1342.8(40.1 \%)$ & $1748.2(48.4 \%)$ \\
\hline $25+$ & $275.4(9.1 \%)$ & $242.9(6.4 \%)$ & $117.7(5.3 \%)$ & $247.0(6.8 \%)$ \\
\hline Total (\%) & 100 & 100 & 100 & 100 \\
\hline \multicolumn{5}{|l|}{ Sexual autonomy } \\
\hline No & $144.3(6.2 \%)$ & $493.5(15.2 \%)$ & $488.8(17.6 \%)$ & $457.5(15.1 \%)$ \\
\hline Yes & 2171.8 (93.0\%) & $2722.9(84.0 \%)$ & $2275.2(81.9 \%)$ & $2489.5(82.4 \%)$ \\
\hline Don't know & $20.0(0.9 \%)$ & $24.7(0.8 \%)$ & $15.1(0.5 \%)$ & $74.0(2.5 \%)$ \\
\hline Total (\%) & 100 & 100 & 100 & 100 \\
\hline \multicolumn{5}{|l|}{ Wealth index } \\
\hline Poorest & $110.4(2.6 \%)$ & $982.4(19.5 \%)$ & $2174.5(49.8 \%)$ & $405.1(8.5 \%)$ \\
\hline Poorer & $349.6(8.1 \%)$ & $1224.3(24.3 \%)$ & 964.5 (22.1\%) & $1022.6(21.4 \%)$ \\
\hline Middle & $555.6(12.9 \%)$ & $1084.3(21.5 \%)$ & $469.6(10.8 \%)$ & $1374.8(28.8 \%)$ \\
\hline Richer & $948.2(21.9 \%)$ & $1031.4(20.5 \%)$ & $431.7(9.9 \%)$ & $1175.2(24.6 \%)$ \\
\hline Richest & $2361.2(54.6 \%)$ & 716.7 (14.2\%) & $327.8(7.5 \%)$ & 796.4 (16.7\%) \\
\hline Total (\%) & 100 & 100 & 100 & 100 \\
\hline \multicolumn{5}{|l|}{ Residence } \\
\hline Urban & 2075.1 (48.0\%) & $798.0(15.8 \%)$ & $654.7(15.0 \%)$ & $1047.5(21.9 \%)$ \\
\hline Rural & 2249.9 (52.0\%) & $4241.0(84.2 \%)$ & $3713.3(85.0 \%)$ & 3726.5 (78.1\%) \\
\hline Total (\%) & 100 & 100 & 100 & 100 \\
\hline \multicolumn{5}{|l|}{ Age at first sex } \\
\hline Not had sex & $606.9(14.1 \%)$ & 715.3 (14.2\%) & $692.6(15.9 \%)$ & $697.5(14.6 \%)$ \\
\hline Below 15 & $578.6(13.4 \%)$ & $1065.5(21.2 \%)$ & $606.2(13.9 \%)$ & 767.5 (16.1\%) \\
\hline
\end{tabular}


Table 2 (continued)

\begin{tabular}{|c|c|c|c|c|}
\hline \multirow[t]{2}{*}{ Characteristics } & \multicolumn{4}{|l|}{ Regions } \\
\hline & $\begin{array}{l}\text { Central } \\
(n=4325)\end{array}$ & $\begin{array}{l}\text { Eastern } \\
(n=5039)\end{array}$ & $\begin{array}{l}\text { Northern } \\
(n=4368)\end{array}$ & $\begin{array}{l}\text { Western } \\
(n=4600)\end{array}$ \\
\hline $15-19$ & $2591.6(60.0 \%)$ & $2966.6(58.9 \%)$ & $2695.4(61.7 \%)$ & $2749.2(57.6 \%)$ \\
\hline $20-24$ & $493.3(11.4 \%)$ & $270.1(5.4 \%)$ & $331.2(7.6 \%)$ & $504.1(10.6 \%)$ \\
\hline $25+$ & $49.5(1.1 \%)$ & $20.6(0.4 \%)$ & $41.6(0.9 \%)$ & $54.8(1.1 \%)$ \\
\hline Total (\%) & 100 & 100 & 100 & 100 \\
\hline \multicolumn{5}{|l|}{ Age at first birth } \\
\hline$\leq 14$ & $231.4(7.5 \%)$ & $312.7(8.4 \%)$ & $221.3(6.7 \%)$ & $249.7(6.9 \%)$ \\
\hline $15-19$ & $1684.0(54.3 \%)$ & $2421.6(64.7 \%)$ & $2055.6(62.6 \%)$ & $1987.4(54.9 \%)$ \\
\hline $20-24$ & $967.2(31.2 \%)$ & $854.5(22.8 \%)$ & $865.2(26.4 \%)$ & $1135.3(31.4 \%)$ \\
\hline $25+$ & $219.4(7.1 \%)$ & $153.2(4.1 \%)$ & $138.9(4.1 \%)$ & $247.7(6.8 \%)$ \\
\hline Total (\%) & 100 & 100 & 100 & 100 \\
\hline \multicolumn{5}{|c|}{ Perception of distance to health facility } \\
\hline A big problem & $1118.1(25.8 \%)$ & 1990.5 (39.5\%) & $2162.2(49.5 \%)$ & $1884.2(39.5 \%)$ \\
\hline Not a big problem & $3206.9(74.2 \%)$ & $3048.5(60.5 \%)$ & $2205(50.5 \%)$ & $2889.8(60.5 \%)$ \\
\hline Total (\%) & 100 & 100 & 100 & 100 \\
\hline \multicolumn{5}{|l|}{ Desire for children } \\
\hline Wants within 2 years & $632.1(14.6 \%)$ & $542.0(10.8 \%)$ & $477.0(10.9 \%)$ & $547.4(11.5 \%)$ \\
\hline Wants after 2 years & $1655.5(38.3 \%)$ & $2049.3(40.7 \%)$ & $1953.7(44.7 \%)$ & $1810.5(37.9 \%)$ \\
\hline Wants, but unsure of timing & $519.2(12.0 \%)$ & $465.3(9.2 \%)$ & $334.6(7.7 \%)$ & $445.1(9.3 \%)$ \\
\hline Undecided & $179.4(4.2 \%)$ & $215.7(4.3 \%)$ & $165.6(3.8 \%)$ & $179.9(3.8 \%)$ \\
\hline Wants no more & $1338.8(31.0 \%)$ & $1766.7(35.1 \%)$ & $1437.2(32.9 \%)$ & $1791.2(37.5 \%)$ \\
\hline Total (\%) & 100 & 100 & 100 & 100 \\
\hline \multicolumn{5}{|l|}{ Number of living children } \\
\hline 0 & $1244.5(28.8 \%)$ & 1.358.3(27.0\%) & $1140.1(26.1 \%)$ & $1172.1(24.6 \%)$ \\
\hline 1 & $693.6(16.0 \%)$ & $615.0(12.2 \%)$ & $584.9(13.4 \%)$ & $667.7(14.0 \%)$ \\
\hline 2 & $619.4(14.3 \%)$ & $609.3(12.1 \%)$ & $531.3(12.2 \%)$ & $653.7(13.7 \%)$ \\
\hline $3+$ & $1767.6(40.9 \%)$ & $2456.5(48.8 \%)$ & $2111.7(48.3 \%)$ & $2280.5(47.8 \%)$ \\
\hline Total (\%) & 100 & 100 & 100 & 100 \\
\hline \multicolumn{5}{|c|}{ Number of children born in the 5 years } \\
\hline 0 & $2111.7(48.8 \%)$ & $2222.3(44.1 \%)$ & $1829.7(41.9 \%)$ & $2117.7(44.4 \%)$ \\
\hline 1 & $1328.7(30.7 \%)$ & $1442.3(28.6 \%)$ & $1464.5(33.5 \%)$ & $1523.3(31.9 \%)$ \\
\hline $2+$ & $884.6(20.5 \%)$ & $1374.4(27.3 \%)$ & $1073.9(24.6 \%)$ & $1133.0(23.7 \%)$ \\
\hline Total (\%) & 100 & 100 & 100 & 100 \\
\hline \multicolumn{5}{|l|}{ Listen to radio } \\
\hline No & $680.6(15.7 \%)$ & $1450.8(28.8 \%)$ & $1550.0(35.5 \%)$ & $1298.8(27.2 \%)$ \\
\hline Yes & $3644.4(84.3 \%)$ & $3588.2(71.2 \%)$ & $2818.1(64.5 \%)$ & $3475.2(72.8 \%)$ \\
\hline Total (\%) & 100 & 100 & 100 & 100 \\
\hline \multicolumn{5}{|l|}{ Watch television } \\
\hline No & $1732.4(40.1 \%)$ & $3793.5(75.3 \%)$ & $3728.7(85.4 \%)$ & $3714.9(77.8 \%)$ \\
\hline Yes & $2592.6(59.9 \%)$ & $1245.6(24.7 \%)$ & $639.3(14.6 \%)$ & $1059.1(22.2 \%)$ \\
\hline Total (\%) & 100 & 100 & 100 & 100 \\
\hline \multicolumn{5}{|c|}{ Reading of newspaper or magazine } \\
\hline No & $2536.9(58.7 \%)$ & $4034.1(80.1 \%)$ & $3890.5(89.1 \%)$ & $4038.2(84.6 \%)$ \\
\hline Yes & $1788.1(41.3 \%)$ & $1005.0(19.9 \%)$ & 477.5 (10.9\%) & 735.8 (15.4\%) \\
\hline Total (\%) & 100 & 100 & 100 & 100 \\
\hline \multicolumn{5}{|l|}{ Employment status } \\
\hline Unemployed & 1310.1 (30.3\%) & $1413.6(28.1 \%)$ & $843.2(19.3 \%)$ & $1320.3(27.7 \%)$ \\
\hline Employed & $3014.9(69.7 \%)$ & $3625.4(71.9 \%)$ & $3524.8(80.7 \%)$ & 3453.7 (72.3\%) \\
\hline
\end{tabular}


Table 2 (continued)

\begin{tabular}{|c|c|c|c|c|}
\hline \multirow[t]{2}{*}{ Characteristics } & \multicolumn{4}{|l|}{ Regions } \\
\hline & $\begin{array}{l}\text { Central } \\
(n=4325)\end{array}$ & $\begin{array}{l}\text { Eastern } \\
(n=5039)\end{array}$ & $\begin{array}{l}\text { Northern } \\
(n=4368)\end{array}$ & $\begin{array}{l}\text { Western } \\
(n=4600)\end{array}$ \\
\hline Total (\%) & 100 & 100 & 100 & 100 \\
\hline
\end{tabular}

Weighted percentage distributions

contraceptive nonuse, they were adopted at the multivariate level of analysis.

\section{Multivariate results}

Table 4 shows results of a binary multivariable logistic regression on contraceptive nonuse by socio-economic and demographic predictors across regions. The results reveal that women's educational level, wealth index, number of living children and number of children born in the last 5 years were the only significant predictors of contraceptive nonuse across regions $(p<0.05)$. The results also demonstrate that respondents' current age, religious affiliation, age at first marriage, respondent's sexual autonomy, age at first sex, age at first birth, desire for children, listening to radio and employment status were only significant predictors of contraceptive nonuse in particular regions. Conversely, residence, perception of distance to health facility, watching television, and reading newspapers or magazines were not predictors of contraceptive nonuse in any of the regions $(p>0.05)$.

Educational level unevenly predicted contraceptive nonuse across regions. In the results, only secondary educational level compared to no educational level was a very strong predictor of contraceptive nonuse across all regions $(p<0.001)$. Increased educational level was associated with lower odds of contraceptive nonuse across regions in comparison with no educational level. In addition, wealth index erratically predicted contraceptive nonuse across regions, although with lower odds of contraceptive nonuse among the poorer, middle, richer and richest women compared with the women in the poorest wealth index; for example, in Central region, the association between contraceptive nonuse and women in the middle and richer wealth index was strong and moderate, respectively unlike in Eastern region where in relationship was moderate.

Results also reveal that there were variations in effect between respondents' number of children born in the last five (5) years and contraceptive nonuse across regions. Central region had a strong association and lower odds of contraceptive nonuse among women who had one child born in the last 5 years prior to the survey compared to women who never had a child born in the last 5 years prior the demographic survey $(p<0.01)$. Unlike Central region, Northern and Western region had a moderate association with lower odds of contraceptive nonuse among women with one birth in the last 5 years prior to the survey, compared to the women with no birth at all in the last 5 years prior the survey $(p<0.01)$.

Religion only predicted contraceptive nonuse in Eastern, Northern and Western region with variations. In Eastern region, religious category of "others" had moderate association and reduced odds of contraceptive nonuse compared with the "Catholics". Also, women in the "Anglican" religion had very association with lower odds of contraceptive nonuse compared with their counterparts the "Catholics" $(p<0.001)$. In addition, results showed that the "Muslim" in Northern region had a strong effect with increased odds of contraceptive nonuse compared with the "Catholics" $(p<0.01)$. And, in the same region, "Pentecostal" with moderate association and lower odds of contraceptive nonuse compared with the "Catholics" $(p<0.05)$.

Besides, results showed that age at first sex was not a determinant of contraceptive nonuse across regions, but a moderate $(p<0.05)$ determinant of contraceptive nonuse in Central and Western region. In Central region, women aged 25 and above were four times more likely not to use contraceptives compared with the women whose age at first sex was below 15 years. In Western region, instead women whose age at first sex was 15-19 had reduced odds of contraceptive nonuse compared to the women whose age at first sex was below the age 15.

\section{Discussion}

The study provided a wider assessment of socio-economic and demographic determinants of contraceptive nonuse across regions in Uganda among women aged 15-49 using the 2016 Uganda Demographic and Health Survey data. Again, amidst differences in effect, the study found educational level, wealth index number of living children and numbers of children born in the last 5 years prior to the demographic survey as the only predictors of contraceptive nonuse across all regions. Respondents' age, religion, age at first marriage, age at first birth, Sexual autonomy, age at first sex, desire for children, listen to radio, and employment status were predictors of contraceptive nonuse in particular regions.

No study in Uganda can justify the differences in effect of women's educational level on contraceptive nonuse 
Table 3 Percentage distribution of socio-economic and demographic characteristics of respondents by contraceptive nonuse across regions

\begin{tabular}{|c|c|c|c|c|c|c|c|c|}
\hline \multirow[t]{3}{*}{ Variables } & \multicolumn{8}{|l|}{ Regions } \\
\hline & \multicolumn{2}{|l|}{ Central } & \multicolumn{2}{|l|}{ Eastern } & \multicolumn{2}{|l|}{ Northern } & \multicolumn{2}{|l|}{ Western } \\
\hline & Non-use & Use & Non-use & Use & Non-use & Use & Non-use & Use \\
\hline \multicolumn{9}{|l|}{ Age } \\
\hline $15-19$ & $679.7(76.1 \%)$ & 213.7 (23.9\%) & $1021.1(79.2 \%)$ & $267.5(20.8 \%)$ & $971.9(87.5 \%)$ & $139.2(12.5 \%)$ & $872.2(85.6 \%)$ & $147.1(14.4 \%)$ \\
\hline $20-24$ & $329.4(33.1 \%)$ & $664.5(66.9 \%)$ & $392.1(38.7 \%)$ & $622.3(61.3 \%)$ & $441.0(52.8 \%)$ & $393.9(47.2 \%)$ & $388.8(41.5 \%)$ & $547.7(58.5 \%)$ \\
\hline $25-29$ & $135.8(17.2 \%)$ & $655.2(82.8 \%)$ & $161.9(21.9 \%)$ & $579.3(78.1 \%)$ & $242.6(36.2 \%)$ & $426.8(63.8 \%)$ & $223.5(27.3 \%)$ & $594.1(72.7 \%)$ \\
\hline $30-34$ & $94.4(16.5 \%)$ & $478.2(83.5 \%)$ & $139.8(21.4 \%)$ & $512.2(78.6 \%)$ & $222.3(35.1 \%)$ & $411.6(64.9 \%)$ & $164.1(23.6 \%)$ & $532.9(76.4 \%)$ \\
\hline $35-39$ & $96.6(19.9 \%)$ & $389.3(80.1 \%)$ & $130.1(25.7 \%)$ & $375.4(74.3 \%)$ & $179.3(39.4 \%)$ & $275.7(60.6 \%)$ & 150.4 (27.0\%) & $406.5(73.0 \%)$ \\
\hline $40-44$ & $77.6(22.9 \%)$ & $261.2(77.1 \%)$ & $151.6(30.9 \%)$ & $338.2(69.1 \%)$ & $184.0(47.7 \%)$ & $201.5(52.3 \%)$ & $146.6(36.1 \%)$ & $259.5(63.9 \%)$ \\
\hline \multirow[t]{2}{*}{$45-49$} & $77.7(31.1 \%)$ & 171.8 (68.9\%) & 156.1 (44.9\%) & $191.4(55.1 \%)$ & $179.7(64.6 \%)$ & $98.6(35.4 \%)$ & $138.6(40.7 \%)$ & $202.1(59.3 \%)$ \\
\hline & \multicolumn{2}{|c|}{$x^{2}=939.3498, p=0.0000$} & \multicolumn{2}{|c|}{$x^{2}=1049.7960, p=0.0000$} & \multicolumn{2}{|c|}{$x^{2}=736.1774, p=0.0000$} & \multicolumn{2}{|c|}{$x^{2}=1006.2228, p=0.0000$} \\
\hline \multicolumn{9}{|c|}{ Educational level } \\
\hline No education & $81.8(35.4 \%)$ & $149.5(64.6 \%)$ & $170.7(48.5 \%)$ & $181.4(51.5 \%)$ & $494.4(65.8 \%)$ & $256.7(34.2 \%)$ & $253(45.4 \%)$ & $304.8(54.6 \%)$ \\
\hline Primary & $652.8(35.1 \%)$ & $1209.7(64.9 \%)$ & $1351.6(43.4 \%)$ & $1764.5(56.6 \%)$ & $1607.9(54.9 \%)$ & $1323.5(45.1 \%)$ & $1326(44.5 \%)$ & $1655.7(55.5 \%)$ \\
\hline Secondary & $581.1(36.0 \%)$ & $1032.2(64.0 \%)$ & $556.5(43.0 \%)$ & $736.6(57.0 \%)$ & $254.3(51.4 \%)$ & $240.9(48.6 \%)$ & $482.6(43.9 \%)$ & $547.2(56.1 \%)$ \\
\hline \multirow[t]{2}{*}{ Higher } & $175.6(28.4 \%)$ & $442.5(71.6 \%)$ & $73.9(26.6 \%)$ & $203.7(73.4 \%)$ & $64.1(33.7 \%)$ & $126.2(66.3 \%)$ & $76.6(29.6 \%)$ & $182.1(70.4 \%)$ \\
\hline & \multicolumn{2}{|c|}{$x^{2}=12.1249, p=0.0995$} & \multicolumn{2}{|c|}{$x^{2}=34.7707, p=0.0007$} & \multicolumn{2}{|c|}{$x^{2}=73.0577, p=0.0000$} & \multicolumn{2}{|c|}{$x^{2}=22.2693, p=0.0008$} \\
\hline \multicolumn{9}{|l|}{ Religious status } \\
\hline Catholics & $476.8(31.6 \%)$ & $1034.2(68.4 \%)$ & $623.4(44.4 \%)$ & $780.5(55.6 \%)$ & $1485(57.4 \%)$ & $1102.1(42.6 \%)$ & $907.3(44.6 \%)$ & $1127.5(55.4 \%)$ \\
\hline Anglican & $379.2(33.3 \%)$ & $759.4(66.7 \%)$ & $794.2(43.0 \%)$ & $1051.4(57.0 \%)$ & $468.7(47.3 \%)$ & $522.2(52.7 \%)$ & $737.3(40.8 \%)$ & $1068.1(59.2 \%)$ \\
\hline Muslim & $261.7(32.9 \%)$ & $532.5(67.1 \%)$ & $342.9(39.6 \%)$ & $532.3(60.4 \%)$ & $269.3(65.7 \%)$ & $140.5(34.3 \%)$ & $92.6(42.4 \%)$ & $126.0(57.6 \%)$ \\
\hline Pentecostal & $313.1(42.2 \%)$ & $429.4(57.8 \%)$ & $353.8(44.3 \%)$ & $445.3(55.7 \%)$ & $183.1(50.8 \%)$ & 177.5 (49.2\%) & $220.1(46.0 \%)$ & $258.0(54.0 \%)$ \\
\hline \multirow{2}{*}{ Others } & $60.5(43.6 \%)$ & $78.3(56.4 \%)$ & $38.5(31.0 \%)$ & $85.8(69.0 \%)$ & $14.6(74.4 \%)$ & $5.0(25.6 \%)$ & $126.9(53.5 \%)$ & 110.2 (46.5\%) \\
\hline & $x^{2}=31.7611, p$ & $=0.0005$ & $x^{2}=12.9586, p=$ & $=0.0870$ & $x^{2}=54.1094, p$ & $=0.0000$ & $x^{2}=17.2144, p=$ & $=0.0204$ \\
\hline Age at first $m$ & age & & & & & & & \\
\hline$\leq 12$ & $17.9(19.9 \%)$ & 72.4 (80.1\%) & $50.4(35.8 \%)$ & $90.2(64.2 \%)$ & $49.8(41.6 \%)$ & $69.8(58.4 \%)$ & $63.0(44.4 \%)$ & $79.0(55.6 \%)$ \\
\hline $13-17$ & $231.0(20.1 \%)$ & 919.9 (79.9\%) & $555.2(30.7 \%)$ & $1255.9(69.3 \%)$ & 712.5 (41.7\%) & 994.3 (58.3\%) & 459.2 (31.1\%) & $1,015.7$ (68.9\%) \\
\hline $18-24$ & 316.4 (20.8\%) & 1203 (79.2\%) & 460.7 (29.2\%) & 1117.8 (70.8\%) & $657.6(49.0 \%)$ & $685.3(51.0 \%)$ & $542.8(31.0 \%)$ & 1,205.4 (69.0\%) \\
\hline $25+$ & 316.4 (19.4\%) & $222.1(80.6 \%)$ & $78.3(32.2 \%)$ & $164.6(67.8 \%)$ & $84.4(47.5 \%)$ & $93.4(52.5 \%)$ & 79.7 (32.3\%) & $167.2(67.7 \%)$ \\
\hline & $x^{2}=0.4413 p=$ & 9561 & $x^{2}=3.5023 p=$ & .4181 & $x^{2}=16.8430$ & $=0.0028$ & $x^{2}=11.1670 p$ & $=0.0394$ \\
\hline Sexual autonor & & & & & & & & \\
\hline No & $44.9(31.1 \%)$ & 99.3 (68.9\%) & 175.7 (35.6\%) & $317.8(64.4 \%)$ & $227.9(46.6 \%)$ & $260.9(53.4 \%)$ & 168.7 (36.9\%) & $288.9(63.1 \%)$ \\
\hline Yes & 405.9 (18.7\%) & $1765.8(81.3 \%)$ & 801.8 (29.5\%) & $1921.1(70.5 \%)$ & 1021.3 (44.9\%) & $1253.9(55.1 \%)$ & 738.6 (29.7\%) & 1750.9 (70.3\%) \\
\hline Don't know & $1.5(7.7 \%)$ & 18.5 (92.3\%) & $6.4(26.0 \%)$ & $18.2(74.0 \%)$ & $4.4(29.4 \%)$ & $10.6(70.6 \%)$ & $16.6(22.5 \%)$ & $57.3(77.5 \%)$ \\
\hline & $x^{2}=15.2060, p$ & 0.0024 & $x^{2}=7.7185, p=$ & 0.0724 & $x^{2}=1.9994, p=$ & 0.4484 & $x^{2}=11.7622, p=$ & $=0.0070$ \\
\hline Wealth quintile & & & & & & & & \\
\hline Poorest & $46.6(42.2 \%)$ & $63.8(57.8 \%)$ & $436.6(44.4 \%)$ & $545.8(55.6 \%)$ & $1348(62.0 \%)$ & 826.4 (38.0\%) & 246.7 (60.9\%) & 158.4 (39.1\%) \\
\hline Poorer & $142.9(40.9 \%)$ & 206.7 (59.1\%) & $530.9(43.4 \%)$ & $693.4(56.6 \%)$ & 480.1 (49.8\%) & 484.5 (50.2\%) & 476.6 (46.9\%) & $543.0(53.1 \%)$ \\
\hline Middle & $191.1(34.4 \%)$ & $364.5(65.6 \%)$ & $484.4(44.7 \%)$ & $599.9(55.3 \%)$ & $221.7(47.2 \%)$ & $247.9(52.8 \%)$ & $610.2(44.4 \%)$ & 764.5 (55.6\%) \\
\hline Richer & $322.2(34.0 \%)$ & $626.0(66.0 \%)$ & 419.5 (40.7\%) & $612.0(59.3 \%)$ & $206.2(47.8 \%)$ & $225.4(52.2 \%)$ & $451.6(38.4 \%)$ & 723.5 (61.6\%) \\
\hline Richest & 788.5 (33.4\%) & $1572.8(66.6 \%)$ & $281.5(39.3 \%)$ & 435.2 (60.7\%) & 164.7 (50.3\%) & $163.1(49.7 \%)$ & $296.0(37.2 \%)$ & $500.3(62.8 \%)$ \\
\hline & $x^{2}=10.5514 p$ & 0.225 & $x^{2}=8.3496 p=$ & .2145 & $x^{2}=77.0443 p .0$ & .0000 & $x^{2}=80.3140 p=$ & $=0.0000$ \\
\hline Residence & & & & & & & & \\
\hline Urban & 675.5 (32.5\%) & $1399.6(67.5 \%)$ & 302.4 (37.9\%) & $495.6(62.1 \%)$ & 359.3 (54.9\%) & $295.3(45.1 \%)$ & 389.1 (37.1\%) & 658.5 (62.9\%) \\
\hline Rural & 815.7 (36.3\%) & $1434.2(63.7 \%)$ & 1850.4 (43.6\%) & $2390.6(56.4 \%)$ & $2061.4(55.5 \%)$ & $1651.9(44.5 \%)$ & $1695.2(45.5 \%)$ & $2031.3(54.5 \%)$ \\
\hline & $x^{2}=6.5424 p=$ & .1226 & $x^{2}=9.0415 p=$ & 0.0170 & $x^{2}=0.0884 p=$ & 0.8895 & $x^{2}=23.1874 p=$ & $=0.0080$ \\
\hline Age at first sex & & & & & & & & \\
\hline Not had sex & $601.4(99.1 \%)$ & $5.6(0.9 \%)$ & 703.9 (98.4\%) & $11.4(1.6 \%)$ & 686.7 (99.2\%) & $5.9(0.9 \%)$ & 694.9 (99.6\%) & $2.5(0.4 \%)$ \\
\hline
\end{tabular}


Table 3 (continued)

\begin{tabular}{|c|c|c|c|c|c|c|c|c|}
\hline \multirow[t]{3}{*}{ Variables } & \multicolumn{8}{|l|}{ Regions } \\
\hline & \multicolumn{2}{|l|}{ Central } & \multicolumn{2}{|l|}{ Eastern } & \multicolumn{2}{|l|}{ Northern } & \multicolumn{2}{|l|}{ Western } \\
\hline & Non-use & Use & Non-use & Use & Non-use & Use & Non-use & Use \\
\hline Below 15 & $134.8(23.3 \%)$ & $443.8(76.7 \%)$ & $341.5(32.0 \%)$ & $723.9(68.0 \%)$ & $276.2(45.6 \%)$ & $330.0(54.4 \%)$ & $287.3(37.4 \%)$ & $480.1(62.6 \%)$ \\
\hline $15-19$ & 597.5 (23.0\%) & $1994.2(77.0 \%)$ & $1009.4(34.0 \%)$ & $1957.2(66.0 \%)$ & $1258.3(46.7 \%)$ & $1437.1(53.3 \%)$ & 917.4 (33.4\%) & $1831.8(66.6 \%)$ \\
\hline $20-24$ & $136.1(27.6 \%)$ & $357.2(72.4 \%)$ & $85.0(31.5 \%)$ & $185.0(68.5 \%)$ & $167.8(50.7 \%)$ & $163.4(49.3 \%)$ & 160.7 (31.9\%) & $343.4(68.1 \%)$ \\
\hline \multirow[t]{2}{*}{$25+$} & $17.5(35.3 \%)$ & $32.0(64.7 \%)$ & $12.4(60.3 \%)$ & $8.2(39.7 \%)$ & $30.6(73.5 \%)$ & $11.0(26.5 \%)$ & $22.5(41.1 \%)$ & $32.3(58.9 \%)$ \\
\hline & \multicolumn{2}{|c|}{$x^{2}=1314.3009 p=0.0000$} & \multicolumn{2}{|c|}{$x^{2}=1064.1139 p=0.0000$} & \multicolumn{2}{|c|}{$x^{2}=651.7632 p=0.0000$} & \multicolumn{2}{|c|}{$x^{2}=1047.4633 p=0.0000$} \\
\hline \multicolumn{9}{|c|}{ Age at first birth } \\
\hline Below 15 & $38.0(16.4 \%)$ & $193.4(83.6 \%)$ & $87.3(27.9 \%)$ & $225.4(72.1 \%)$ & $82.1(37.1 \%)$ & $139.2(62.9 \%)$ & $97.6(39.1 \%)$ & $152.1(60.9 \%)$ \\
\hline $15-19$ & $307.2(18.2 \%)$ & $1376.8(81.8 \%)$ & $698.5(28.8 \%)$ & $1723.2(71.2 \%)$ & $832.3(40.5 \%)$ & $1223.4(59.5 \%)$ & $536.2(27.0 \%)$ & $1451.2(73.0 \%)$ \\
\hline $20-24$ & $187.0(19.3 \%)$ & $780.3(80.7 \%)$ & $253.1(29.6 \%)$ & $601.4(70.4 \%)$ & $431.5(49.9 \%)$ & $433.6(50.1 \%)$ & 350.7 (30.9\%) & 784.7 (69.1\%) \\
\hline \multirow{2}{*}{$25+$} & $56.5(25.8 \%)$ & $162.8(74.2 \%)$ & $55.2(36.0 \%)$ & $98.0(64.0 \%)$ & $72.9(52.5 \%)$ & $66.0(47.5 \%)$ & $100.2(40.5 \%)$ & $147.4(59.5 \%)$ \\
\hline & \multicolumn{2}{|c|}{$x^{2}=8.2373 p=0.1196$} & \multicolumn{2}{|c|}{$x^{2}=3.9420 p=0.3506$} & \multicolumn{2}{|c|}{$x^{2}=30.1347 p=0.0000$} & \multicolumn{2}{|c|}{$x^{2}=31.8334 p=0.0001$} \\
\hline \multicolumn{9}{|c|}{ Perception of distance to health facility } \\
\hline A big problem & $360.3(32.2 \%)$ & $757.8(67.8 \%)$ & $861.4(43.3 \%)$ & $1129.1(56.7 \%)$ & $1163.2(53.8 \%)$ & $999.0(46.2 \%)$ & $845.0(44.9 \%)$ & $1039.1(55.1 \%)$ \\
\hline \multirow{2}{*}{$\begin{array}{l}\text { Not a big } \\
\text { problem }\end{array}$} & $1130.9(35.3 \%)$ & $2076.0(64.7 \%)$ & $1291.4(42.4 \%)$ & $1757.1(57.6 \%)$ & $1257.5(57.0 \%)$ & $948.3(43.0 \%)$ & $1239.2(42.9 \%)$ & $1650.6(57.1 \%)$ \\
\hline & \multicolumn{2}{|c|}{$x^{2}=3.3924 p=0.1179$} & \multicolumn{2}{|c|}{$x^{2}=0.4099 p=0.5777$} & \multicolumn{2}{|c|}{$x^{2}=4.5650 p=0.1119$} & $x^{2}=1.7954 p$ & 951 \\
\hline Desire for childre & & & & & & & & \\
\hline $\begin{array}{l}\text { Wants within } \\
2 \text { years }\end{array}$ & $199.0(31.5 \%)$ & $433.1(68.5 \%)$ & $200.4(37.0 \%)$ & $341.6(63.0 \%)$ & $289.9(60.8 \%)$ & $187.1(39.2 \%)$ & $274.4(50.1 \%)$ & $273.0(49.9 \%)$ \\
\hline $\begin{array}{l}\text { Wants after } \\
2 \text { years }\end{array}$ & $571.1(34.5 \%)$ & $1084.5(65.5 \%)$ & $941.3(45.9 \%)$ & $1107.9(54.1 \%)$ & $1092.1(55.9 \%)$ & $861.6(44.1 \%)$ & $847.3(46.8 \%)$ & $963.2(53.2 \%)$ \\
\hline $\begin{array}{l}\text { Wants, but } \\
\text { unsure of } \\
\text { timing }\end{array}$ & $348.2(67.1 \%)$ & $170.9(32.9 \%)$ & $351.6(74.6 \%)$ & $113.8(24.4 \%)$ & $303.0(90.5 \%)$ & $31.7(9.5 \%)$ & $351.3(78.9 \%)$ & $93.8(21.1 \%)$ \\
\hline Undecided & $85.3(47.6 \%)$ & $94.1(52.4 \%)$ & 131.4 (60.9\%) & $84.3(39.1 \%)$ & 102.5 (61.9\%) & $63.0(38.1 \%)$ & $80.1(44.5 \%)$ & $99.8(55.5 \%)$ \\
\hline Wants no more & $287.6(21.5 \%)$ & $1051.2(78.5 \%)$ & $528.1(29.9 \%)$ & $1238.6(70.1 \%)$ & $633.2(44.1 \%)$ & 803.9 (55.9\%) & $531.2(29.7 \%)$ & 1260 (70.3\%) \\
\hline & $x^{2}=360.4156 p$ & $=0.0000$ & $x^{2}=368.8890 p$ & $=0.0000$ & $x^{2}=250.5904 p$ & $=0.0000$ & $x^{2}=384.5077$ & $=0.0000$ \\
\hline Number of living & children & & & & & & & \\
\hline 0 & 919.4 (73.9\%) & 325.1 (26.1\%) & 1085.6 (79.9\%) & 272.7 (20.1\%) & $1037.8(91.0 \%)$ & $102.3(9.0 \%)$ & 1015.7 (86.7\%) & 156.4 (13.3\%) \\
\hline 1 & 195.4 (28.1\%) & 498.1 (71.8\%) & $281.9(45.8 \%)$ & 333.1 (54.2\%) & $337.2(57.7 \%)$ & $247.6(42.3 \%)$ & 293.6 (44.0\%) & 374.1 (56.0\%) \\
\hline 2 & $97.3(15.7 \%)$ & $522.1(84.3 \%)$ & 162.7 (26.7\%) & 446.6 (73.3\%) & $221.1(41.6 \%)$ & 310.3 (58.4\%) & 199.8 (30.6\%) & $454.0(69.4 \%)$ \\
\hline $3+$ & 279.1 (15.8\%) & $1488.6(84.2 \%)$ & 622.7 (25.4\%) & 1834 (74.6\%) & 824.6 (39.1\%) & $1287.1(60.9 \%)$ & $575.2(25.2 \%)$ & $1705.3(74.8 \%)$ \\
\hline & $x^{2}=1237.2615$ & $p=0.0000$ & $x^{2}=1137.4900$ & $=0.0000$ & $x^{2}=856.3612 p$ & $=0.0000$ & $x^{2}=1241.7939$ & $p=0.0000$ \\
\hline Number of chil & en born in the las & 5 years & & & & & & \\
\hline 0 & $1104.7(52.3 \%)$ & $1007.0(47.7 \%)$ & 1351.0 (60.8\%) & 871.4 (39.2\%) & $1364.3(74.6 \%)$ & $465.3(25.4 \%)$ & 1306.7 (61.7\%) & 811.0 (38.3\%) \\
\hline 1 & $218.0(16.4 \%)$ & 1110.8 (83.6\%) & $391.8(27.2 \%)$ & $1050.6(72.8 \%)$ & $568.5(38.8 \%)$ & 896.0 (61.2\%) & $388.0(25.5 \%)$ & $1135.3(74.5 \%)$ \\
\hline $2+$ & $168.6(19.1 \%)$ & 716.0 (80.9\%) & 410.1 (29.8\%) & 964.3 (70.2\%) & $487.9(45.4 \%)$ & $585.9(54.6 \%)$ & 389.5 (34.4\%) & 743.5 (65.6\%) \\
\hline & $x^{2}=582.6405 p$ & $\leq 0.001$ & $x^{2}=532.4315 p$ & $\leq 0.001$ & $x^{2}=478.2326 p$ & $\leq 0.001$ & $x^{2}=524.9012 p$ & $\leq 0.001$ \\
\hline Listen to radio & & & & & & & & \\
\hline No & $281.0(41.3 \%)$ & 399.6 (58.7\%) & 688.4 (47.5\%) & 762.4 (52.5\%) & $927.0(59.8 \%)$ & $623.0(40.2 \%)$ & 732.1 (56.4\%) & $566.7(43.6 \%)$ \\
\hline Yes & $1210.2(33.2 \%)$ & $2434.2(66.8 \%)$ & $1464.4(40.8 \%)$ & $2123.8(59.2 \%)$ & $1493.8(53.0 \%)$ & $1324.3(47.0 \%)$ & 1352.1 (38.9\%) & $2123.0(61.1 \%)$ \\
\hline & $x^{2}=16.6029 p=$ & $=0.0020$ & $x^{2}=18.6168 p=$ & 0.0001 & $x^{2}=18.7018 p=$ & $=0.0021$ & $x^{2}=117.1477 p$ & $=0.0000$ \\
\hline Watch television & & & & & & & & \\
\hline No & $634.1(36.6 \%)$ & $1098.3(63.4 \%)$ & 1640.6 (43.3\%) & $2152.9(56.7 \%)$ & $2071.9(55.6 \%)$ & $1656.8(44.4 \%)$ & 1663.9 (44.8\%) & $2051.0(55.2 \%)$ \\
\hline Yes & 857.1 (33.1\%) & 1735.5 (66.9\%) & $512.2(41.1 \%)$ & 733.4 (58.9\%) & 348.8 (54.6\%) & 290.5 (45.5\%) & 420.3 (39.7\%) & $638.8(60.3 \%)$ \\
\hline & $x^{2}=5.7687 p=$ & 0.0621 & $x^{2}=1.7329 p=$ & .2971 & $x^{2}=0.2241 p=$ & 0.6942 & $x^{2}=8.7283 p=$ & 0.0346 \\
\hline Reading of new & aper or magazin & & & & & & & \\
\hline No & $880.2(34.7 \%)$ & $1656.7(65.3 \%)$ & $1737.3(43.1 \%)$ & $2296.8(56.9 \%)$ & 2166.5 (55.7\%) & $1724.0(44.3 \%)$ & $1773.7(43.9 \%)$ & $2264.6(56.1 \%)$ \\
\hline
\end{tabular}


Table 3 (continued)

\begin{tabular}{|c|c|c|c|c|c|c|c|c|}
\hline \multirow[t]{3}{*}{ Variables } & \multicolumn{8}{|l|}{ Regions } \\
\hline & \multicolumn{2}{|l|}{ Central } & \multicolumn{2}{|l|}{ Eastern } & \multicolumn{2}{|l|}{ Northern } & \multicolumn{2}{|l|}{ Western } \\
\hline & Non-use & Use & Non-use & Use & Non-use & Use & Non-use & Use \\
\hline \multirow[t]{2}{*}{ Yes } & $611.0(34.2 \%)$ & $1177.1(65.8 \%)$ & $415.5(41.4 \%)$ & $589.4(58.6 \%)$ & $254.2(53.2 \%)$ & $223.3(46.8 \%)$ & $310.6(42.2 \%)$ & $425.2(57.8 \%)$ \\
\hline & \multicolumn{2}{|c|}{$x^{2}=0.1270 p=0.8192$} & \multicolumn{2}{|c|}{$x^{2}=0.9696 p=0.4593$} & \multicolumn{2}{|c|}{$x^{2}=1.0307 p=0.4541$} & \multicolumn{2}{|c|}{$x^{2}=0.7420 p=0.4803$} \\
\hline \multicolumn{9}{|c|}{ Employment status } \\
\hline Unemployed & $611.3(46.7 \%)$ & $698.8(53.3 \%)$ & $847.4(60.0 \%)$ & $566.2(40.0 \%)$ & $630.6(74.8 \%)$ & $212.5(25.2 \%)$ & $842.1(63.8 \%)$ & $478.3(36.2 \%)$ \\
\hline \multirow[t]{2}{*}{ Employed } & $879.9(29.2 \%)$ & $2135(70.8 \%)$ & $1305.4(36.0 \%)$ & $2320.1(64.0 \%)$ & $1790.1(50.8 \%)$ & $1734.7(49.2 \%)$ & $1242.2(36.0 \%)$ & $2211.5(64.0 \%)$ \\
\hline & \multicolumn{2}{|c|}{$x^{2}=123.4241 p=0.0000$} & \multicolumn{2}{|c|}{$x^{2}=238.2390 p=0.0000$} & \multicolumn{2}{|c|}{$x^{2}=158.7397 p=0.0000$} & \multicolumn{2}{|c|}{$x^{2}=300.3493 p=0.0000$} \\
\hline
\end{tabular}

Non-use-never used or tried using a contraceptive

Use-Ever used or tried using a contraceptive

across all regions. Although, studies done in Uganda and elsewhere generally associate less schooling of women to contraceptive nonuse [28, 33-37]; linked to lack of knowledge, fatalism, and lack of contraceptive access $[34,38]$. Nevertheless, promotion of women's educational advancements to at least secondary level across regions should be advanced because this could expedite on contraceptive utilization across all regions in Uganda. However, more research needs to be directed towards understanding these variations in effect of the same variably on contraceptive nonuse across regions.

Wealth index variably predicted contraceptive nonuse across regions as explained above. However, no study can clarify these variations in impact of wealth index on women's contraceptive nonuse across all regions. Although, numerous studies have indicated that women in higher wealth index are associated with reduced odds of contraceptive nonuse compared to their counterparts the poorest women [2, 33, 37]. This kind of behavioural pattern has been associated to poverty among women in developing countries [38,39]. Therefore, there is need to advance research towards understanding these variations in effect of wealth index on contraceptive nonuse across all regions.

Further, respondents' number of living children differently predicted contraceptive nonuse across regions as earlier noted. Remarkably, no study in Uganda explains this result; although, studies elsewhere indicate that women with one, or more children have a higher chance of limiting child bearing compared to the women with no children who want to have a child [10, 40-44]. However, more research should be directed towards understanding the effect of respondents' number of living children on contraceptive nonuse in the four regions.

Besides that, there have also not been studies that justify the variance in effect in association of respondents' births in the last 5 years prior to the demographic health survey on contraceptive nonuse across regions of Uganda. Although, a related study among young women in Uganda revealed that women who had a birth in the last 5 years prior to the survey were five times more likely to use contraceptives compared to those who had never had a birth [45]. Nonetheless, extensive studies ought to be done to comprehensively understand the study finding across regions.

Contraceptive nonuse varied by women's age in particularly Eastern Northern and Western region. Somewhat related with the study finding, a non-disintegrated study by region in Uganda revealed that contraceptive use among young women was low [45]; which could be associated to cost, fear, and cultural barriers [46, 47]. In addition, studies indicate that women aged 45-49 older aged are generally expected to have achieved their desired number of children; therefore, are associated with infrequent sexual intercourse, menopause, and sometimes lost interest for sex [48, 49]. Similarly, it is believed that women who are above the age of 25 have attained their fertility desires and therefore more likely to use contraceptives [48]. On the contrary, a study revealed that women aged 35-39 were more likely than women aged 25-29 to use contraceptives [50]. Conversely, investigations should be directed towards understanding extensively the findings of the study.

Religion differently predicted contraceptive nonuse in particularly Eastern, Northern and Western region. Importantly, there is scarce literature to justify the study findings. However, studies elsewhere indicate that lower odds of contraceptive nonuse among the Anglican and Pentecostal religion could be attributed to the absence of restrictions in contraceptive use compared to the Catholic religion that prohibits the use of contraceptives [51, 52]. Further, results show that Muslim women in Northern region had greater odds of contraceptive nonuse compared to the Catholics; this study finding contradicts 
Table 4 Results from a multivariate logistic regression on contraceptive nonuse and women's demographic and socioeconomic factors across all regions (UDHS 2016)

\begin{tabular}{|c|c|c|c|c|c|c|c|c|}
\hline \multirow[t]{3}{*}{ Variables } & \multicolumn{8}{|c|}{ Regions } \\
\hline & \multicolumn{2}{|c|}{ Central } & \multicolumn{2}{|l|}{ Eastern } & \multicolumn{2}{|c|}{ Northern } & \multicolumn{2}{|l|}{ Western } \\
\hline & OR & $95 \% \mathrm{Cl}$ & OR & $95 \% \mathrm{Cl}$ & OR & $95 \% \mathrm{Cl}$ & $\overline{\mathrm{OR}}$ & $95 \% \mathrm{Cl}$ \\
\hline \multicolumn{9}{|l|}{ Age } \\
\hline $15-19^{\mathrm{a}}$ & 1.00 & & 1.00 & & 1.00 & & 1.00 & \\
\hline $20-24$ & 0.74 & $0.36-1.51$ & 0.71 & $0.47-1.07$ & 0.70 & $0.44-1.12$ & $0.56^{*}$ & $0.32-0.96$ \\
\hline $25-29$ & 0.58 & $0.25-1.28$ & $0.56^{*}$ & $0.33-0.94$ & 0.68 & $0.39-1.16$ & 0.60 & $0.33-1.09$ \\
\hline $30-34$ & 0.64 & $0.26-1.55$ & 0.68 & $0.39-1.18$ & 0.66 & $0.37-1.18$ & 0.69 & $0.36-1.31$ \\
\hline $35-39$ & 1.39 & $0.55-3.55$ & 0.89 & $0.48-1.62$ & 0.80 & $0.43-1.49$ & 1.10 & $0.55-2.20$ \\
\hline $40-44$ & 1.10 & $0.39-3.08$ & 1.13 & $0.59-2.12$ & 1.15 & $0.58-2.24$ & 2.01 & $0.97-4.16$ \\
\hline $45-49$ & 2.29 & $0.77-6.79$ & 1.99 & $0.96-4.11$ & $2.13^{*}$ & $11.00-4.54$ & $2.48^{*}$ & $1.12-5.48$ \\
\hline \multicolumn{9}{|l|}{ Educational level } \\
\hline No education ${ }^{\mathrm{a}}$ & 1.00 & & 1.00 & & 1.00 & & 1.00 & \\
\hline Primary & $0.52^{* *}$ & $0.32-0.84$ & $0.44^{* * *}$ & $0.32-0.61$ & $0.45^{* * *}$ & $0.35-0.57$ & $0.67^{* *}$ & $0.51-0.88$ \\
\hline Secondary & $0.28^{* * *}$ & $0.15-0.49$ & $0.34^{* * *}$ & $0.21-0.51$ & $0.36^{* * *}$ & $0.23-0.55$ & $0.46^{* * *}$ & $0.31-0.69$ \\
\hline Higher & $0.28^{* * *}$ & $0.13-0.62$ & $0.15^{* * *}$ & $0.07-0.33$ & $0.20^{* * *}$ & $0.16-0.56$ & 0.58 & $0.33-1.12$ \\
\hline \multicolumn{9}{|l|}{ Religion } \\
\hline Catholics $^{\mathrm{a}}$ & 1.00 & & 1.00 & & 1.00 & & 1.00 & \\
\hline Anglican & 0.88 & $0.61-1.27$ & 1.09 & $0.86-1.38$ & $0.56^{* * *}$ & $0.44-0.71$ & $0.75^{*}$ & $0.60-0.94$ \\
\hline Muslim & 0.88 & $0.58-1.34$ & 1.04 & $0.76-1.42$ & $1.70^{* *}$ & $1.19-2.41$ & 0.77 & $0.46-1.28$ \\
\hline Pentecostal & 1.06 & $0.70-1.62$ & 1.08 & $0.81-1.47$ & $0.69^{*}$ & $0.49-0.97$ & 1.16 & $0.84-1.61$ \\
\hline Others & 1.46 & $0.63-3.39$ & $0.47^{*}$ & $0.24-0.94$ & 1.21 & $0.43-3.38$ & $1.57^{*}$ & $1.05-2.34$ \\
\hline \multicolumn{9}{|c|}{ Age at first marriage } \\
\hline$\leq 12^{\mathrm{a}}$ & 1.00 & & 1.00 & & 1.00 & & 1.00 & \\
\hline $13-17$ & 0.76 & $0.32-1.78$ & 0.72 & $0.38-1.34$ & 1.43 & $0.79-2.60$ & 0.62 & $0.36-1.07$ \\
\hline $18-24$ & 0.81 & $0.33-1.96$ & 0.79 & $0.41-1.52$ & 1.64 & $0.89-3.03$ & $0.55^{*}$ & $0.31-0.98$ \\
\hline $25+$ & 0.46 & $0.16-1.34$ & 0.88 & $0.42-1.84$ & 1.35 & $0.64-2.85$ & 0.48 & $0.23-1.02$ \\
\hline \multicolumn{9}{|c|}{ Sexual autonomy } \\
\hline $\mathrm{No}^{\mathrm{a}}$ & 1.00 & & 1.00 & & 1.00 & & 1.00 & \\
\hline Yes & $0.52^{* *}$ & $0.31-0.85$ & 0.87 & $0.68-1.11$ & 1.09 & $0.85-1.39$ & 0.80 & $0.62-1.04$ \\
\hline Don't know & 0.24 & $0.03-1.64$ & 0.74 & $0.21-2.66$ & 0.44 & $0.11-1.84$ & 0.51 & $0.23-1.12$ \\
\hline \multicolumn{9}{|l|}{ Wealth index } \\
\hline Poorest $\mathrm{t}^{\mathrm{a}}$ & 1.00 & & 1.00 & & 1.00 & & 1.00 & \\
\hline Poorer & 0.65 & $0.28-1.27$ & 0.94 & $0.73-1.21$ & $0.51^{* * *}$ & $0.40-0.65$ & $0.55^{* *}$ & $0.37-0.80$ \\
\hline Middle & $0.34^{* *}$ & $0.15-0.68$ & 0.91 & $0.68-1.22$ & $0.33^{* * *}$ & $0.23-0.47$ & $0.47^{* * *}$ & $0.32-0.69$ \\
\hline Richer & $0.42^{*}$ & $0.19-0.81$ & $0.72^{*}$ & $0.52-0.99$ & $0.37^{* * *}$ & $0.25-0.54$ & $0.37^{* * *}$ & $0.25-0.56$ \\
\hline Richest & 0.50 & $0.20-0.97$ & $0.58^{*}$ & $0.35-0.96$ & $0.42^{* *}$ & $0.24-0.72$ & $0.27^{* * *}$ & $0.16-0.46$ \\
\hline \multicolumn{9}{|l|}{ Residence } \\
\hline$U_{r b a n}^{a}$ & 1.00 & & 1.00 & & 1.00 & & 1.00 & \\
\hline Rural & 1.38 & $0.94-2.04$ & 1.40 & $0.97-2.03$ & 0.99 & $0.72-1.39$ & 1.34 & 0.99-1.82 \\
\hline \multicolumn{9}{|l|}{ Age at first sex } \\
\hline Below $15^{a}$ & 1.00 & & 1.00 & & 1.00 & & 1.00 & \\
\hline $15-19$ & 1.05 & $0.66-1.66$ & 0.12 & $0.87-1.46$ & 0.92 & $0.70-1.22$ & $0.74^{*}$ & $0.55-0.98$ \\
\hline $20-24$ & 1.40 & $0.70-2.79$ & 1.79 & $0.46-1.98$ & 0.73 & $0.47-1.15$ & 0.76 & $0.49-1.16$ \\
\hline $25+$ & $4.01^{*}$ & $1.20-13.40$ & 4.36 & $0.87-21.73$ & 2.54 & $0.67-9.60$ & 0.46 & $0.17-1.22$ \\
\hline \multicolumn{9}{|l|}{ Age at first birth } \\
\hline Below $15^{a}$ & 1.00 & & 1.00 & & 1.00 & & 1.00 & \\
\hline $15-19$ & 1.45 & $0.66-3.20$ & 0.97 & $0.62-1.52$ & 0.87 & $0.57-1.34$ & 0.71 & $0.44-1.16$ \\
\hline $20-24$ & 2.29 & $0.94-5.57$ & 1.18 & $0.70-1.98$ & 1.31 & $0.81-2.13$ & 0.99 & $0.58-1.73$ \\
\hline
\end{tabular}


Table 4 (continued)

\begin{tabular}{|c|c|c|c|c|c|c|c|c|}
\hline \multirow[t]{3}{*}{ Variables } & \multicolumn{8}{|c|}{ Regions } \\
\hline & \multicolumn{2}{|l|}{ Central } & \multicolumn{2}{|c|}{ Eastern } & \multicolumn{2}{|c|}{ Northern } & \multicolumn{2}{|l|}{ Western } \\
\hline & OR & $95 \% \mathrm{Cl}$ & OR & $95 \% \mathrm{Cl}$ & OR & $95 \% \mathrm{Cl}$ & $\overline{\mathrm{OR}}$ & $95 \% \mathrm{Cl}$ \\
\hline $25+$ & $3.58^{*}$ & $1.28-10.03$ & 1.35 & $0.61-3.01$ & 1.16 & $0.53-2.52$ & 1.25 & $0.62-2.53$ \\
\hline \multicolumn{9}{|c|}{ Perception of distance to health facility } \\
\hline A big problem ${ }^{a}$ & 1.00 & & 1.00 & & 1.00 & & 1.00 & \\
\hline Not a big problem & 0.88 & $0.64-1.22$ & 0.94 & $0.77-1.14$ & 1.08 & $0.89-1.31$ & 1.01 & $0.83-1.24$ \\
\hline \multicolumn{9}{|l|}{ Desire for children } \\
\hline Wants within 2 years ${ }^{\mathrm{a}}$ & 1.00 & & 1.00 & & 1.00 & & 1.00 & \\
\hline Wants after 2 years & 1.29 & $0.84-1.99$ & 0.87 & $0.63-1.19$ & 0.78 & $0.56-1.07$ & $0.54^{* * *}$ & $0.39-0.74$ \\
\hline Wants, but unsure of timing & 1.47 & $0.57-3.78$ & 1.89 & $0.89-3.98$ & 0.81 & $0.25-2.65$ & 0.79 & $0.35-1.75$ \\
\hline Undecided & 1.59 & $0.62-4.11$ & $1.87^{*}$ & $1.05-3.34$ & 0.59 & $0.30-1.18$ & 0.97 & $0.57-1.67$ \\
\hline Wants no more & 0.78 & $0.45-1.35$ & 0.77 & $0.54-1.11$ & 0.74 & $0.52-1.05$ & $0.38^{* * *}$ & $0.27-0.54$ \\
\hline \multicolumn{9}{|l|}{ Number of living children } \\
\hline $0^{\mathrm{a}}$ & 1.00 & & 1.00 & & 1.00 & & 1.00 & \\
\hline 1 & $0.15^{* *}$ & $0.43-0.49$ & 1.02 & $0.41-2.46$ & 0.44 & $0.14-1.35$ & 1.12 & $0.44-2.86$ \\
\hline 2 & $0.04^{* * *}$ & $0.01-0.14$ & $0.36^{*}$ & $0.22-1.37$ & $0.17^{* *}$ & $0.06-0.53$ & 0.39 & $0.15-1.02$ \\
\hline $3+$ & $0.03^{* * *}$ & $0.00-0.11$ & $0.24^{* *}$ & $0.15-0.96$ & $0.10^{* * *}$ & $0.03-0.32$ & $0.20^{* *}$ & $0.08-0.55$ \\
\hline \multicolumn{9}{|c|}{ Number of children born in the last 5 years } \\
\hline 0 & 1.00 & & 1.00 & & 1.00 & & 1.00 & \\
\hline 1 & $0.53^{* *}$ & $0.33-0.84$ & 0.77 & $0.56-1.07$ & $0.68^{*}$ & $0.50-0.92$ & $0.71^{*}$ & $0.52-0.96$ \\
\hline $2+$ & 1.26 & $0.77-2.06$ & $1.64^{* *}$ & $1.17-2.29$ & 1.25 & $0.88-1.78$ & $2.32^{* * *}$ & $1.65-3.25$ \\
\hline \multicolumn{9}{|l|}{ Listen to radio } \\
\hline $\mathrm{No}^{\mathrm{a}}$ & 1.00 & & 1.00 & & 1.00 & & 1.00 & \\
\hline Yes & 0.76 & $0.52-1.13$ & 0.90 & $0.73-1.12$ & 0.95 & $0.78-1.17$ & $0.63^{* * *}$ & $0.50-0.80$ \\
\hline \multicolumn{9}{|l|}{ Watch television } \\
\hline $\mathrm{No}^{\mathrm{a}}$ & 1.00 & & 1.00 & & 1.00 & & 1.00 & \\
\hline Yes & 0.75 & $0.51-1.09$ & 1.18 & $0.87-1.58$ & 1.17 & $0.85-1.61$ & 0.81 & $0.60-1.10$ \\
\hline \multicolumn{9}{|c|}{ Reading of newspaper or magazine } \\
\hline $\mathrm{No}^{\mathrm{a}}$ & 1.00 & & 1.00 & & 1.00 & & 1.00 & \\
\hline Yes & 0.74 & $0.51-1.08$ & 1.00 & $0.73-1.37$ & 0.74 & $0.50-1.12$ & 0.82 & $0.56-1.19$ \\
\hline \multicolumn{9}{|l|}{ Employment status } \\
\hline Unemployed $^{\mathrm{a}}$ & 1.00 & & 1.00 & & 1.00 & & 1.00 & \\
\hline Employed & 1.04 & $0.73-1.47$ & $0.78^{*}$ & $0.61-0.99$ & 0.88 & $0.67-1.15$ & $0.75^{*}$ & $0.58-0.96$ \\
\hline
\end{tabular}

OR odds Ratio, Cl Confidence interval

${ }^{*} p$ value $<0.05$ (moderate), ${ }^{* *} p$ value $<0.01$ (strong), ${ }^{* * *} p$ value $<0.001$ (very strong)

a Reference category

with studies that reveal permissiveness of Islam religion in matters of contraceptive use [53, 54]. Conversely, studies into the variations in impact of religion on contraceptive nonuse should be done in the specific regions.

Age at first marriage predicted contraceptive nonuse in only Western region. In regard to the finding, there has not been any study that can explain this result. Although contrary to the finding, a related study in Uganda did not find any association between age at first marriage and contraceptive use [28]. In support of the result, a study found that older age at first marriage was linked to contraceptive nonuse compared to young age at first marriage [41]. This could be because young ages are usually associated with vulnerability that could easily culminate into contraceptive nonuse $[55,56]$. There is therefore need to research into the association between contraceptive nonuse and age at first marriage of 18-24 in Western region.

Sexual autonomy predicted contraceptive nonuse in only Central region. There are no studies that justify this particular effect of sexual autonomy on contraceptive nonuse. However, the outcome could be attributed 
to empowerment, as this makes them have liberty over their bodies and even make knowledgeable choices pertaining contraception uptake [35, 57-60]. Therefore, to comprehensively understand this effect in Central region, advanced research should be done.

Furthermore, results revealed that age at first sex predicted contraceptive nonuse in only Central and Western region. Conversely, there has not been any study to justify this result. The findings in particularly Central and Western region demonstrate that this outcome could be associated with them being in stable relationships unlike their counterparts younger ages [61, 62].

Besides that, the desire for children by respondents' differently predicted contraceptive nonuse in particularly Eastern and Western region. Notably, no study has been undertaken to provide an explanation for this result. Nevertheless, the study finding in Western region resonates with numerous studies [33, 42, 63]. In addition, results in Eastern region are in agreement with a study done by Ahmed Zohirul Islam [64]. However, studies towards holistically understanding the study findings need to be undertaken in the regions.

Listening to radio predicted contraceptive nonuse among women in only Western region. This study could not overtly associate listenership to radio among women in the region. Although, studies non-separated by region suggest that radio is a passage of contraceptive messages and therefore influences contraceptive nonuse [65-67]. However, inquiries should be directed towards understanding the study finding in Western region.

Employment status was a predictor of contraceptive nonuse in Eastern and Western region. Remarkably, no study has been done to explain this study finding. However, non-disintegrated studies suggest that employed women have reduced odds of contraceptive nonuse compared to the unemployed women [25, 33, 42]. This has been linked to the ability to control and make autonomous decisions [25, 68, 69]. Conversely, investigations should be undertaken to understand the impact of employment on contraceptive nonuse in the specific regions.

Nevertheless, this study was limited due to impossibility of determining direction of connectedness of relationships between contraceptive nonuse and socio-economic and demographic variables due to the cross-sectional nature of the data.

\section{Conclusions}

This study has identified four major predictors of contraceptive nonuse across all the regions of Uganda among women aged 15-49. Foremost, educational level, number of living children, wealth index and children born in the last 5 years prior to the survey erratically predicted contraceptive nonuse across all the regions. For particular regions; age, religion, age at first marriage, Sexual autonomy, age at first sex, age at first birth, desire for children, listening to radio, and employment status were predictors of contraceptive nonuse in particular regions of the country. However, residence, perception of distance to health facility, watching television, and reading newspapers or magazines did not predict contraceptive nonuse at all.

Therefore, for Uganda to address the challenges of contraceptive nonuse; deliberate efforts by government and stakeholders need to focus on understanding regional differences and effects of the factors that are associated with contraceptive nonuse. To this end, girl child education should be strengthened to enable completion of higher educational level. For particular regions, this study highlights the need by government and stakeholders to advance academic research in understanding the effect of these predictors on contraceptive nonuse. For example, to understand factors associated with the variations in effect of religion, desire for children, wealth index, age, age at first sex and number of living children on contraceptive nonuse across the regions. This study as well points at the need for researchers to understand the associations and magnitude between contraceptive nonuse and employment status in Eastern and Western region; contraceptive nonuse and radio listening in Western region; contraceptive nonuse and age at first sex in Central and Western region; contraceptive nonuse and age at first birth in Central region; contraceptive nonuse and age at first marriage in Western region; and non-se of contraceptives and the ability to refuse sex in Central region.

\section{Acknowledgements}

The author appreciates Measure DHS for offering him the permission to use 2016 UDHS data.

\section{Authors' contributions}

All authours read and approved the final manuscript

Funding

The author had no funding to the report.

Availability of data and materials

DHS data is available to the public domain through Measure DHS website: https://dhsprogram.com/data/available-datasets.cfm.

Ethics approval and consent to participate

Authorization to utilize the data set was obtained from Measure DHS.

Consent to publish

Not applicable.

Competing interests

Not applicable. 
Received: 28 April 2020 Accepted: 11 December 2020

Published online: 17 December 2020

\section{References}

1. United Nations, Department of Economic and Social Affairs PD. Contraceptive use by method 2019. Data Booklet (ST/ESA/SER.A/435). 2019. https://doi.org/10.18356/1bd58a10-en.

2. Ba DM, Ssentongo P, Agbese E, Kjerulff KH. Prevalence and predictors of contraceptive use among women of reproductive age in 17 sub-Saharan African countries: a large population-based study. Sex Reprod Healthc. 2019:21:26-32.

3. Bongaarts J. WHO, UNICEF, UNFPA, World Bank Group, and United Nations population division trends in maternal mortality: 1990 to 2015 Geneva: World Health Organization, 2015. Popul Dev Rev. 2016;42:726.

4. Bamgboye EA, Ajayi I. Changing patterns of unmet needs for family planning among women of reproductive age in Nigeria. Afr J Reprod Health. 2016;20:127-35.

5. Hubacher D, Mavranezouli I, McGinn E. Unintended pregnancy in sub-Saharan Africa: magnitude of the problem and potential role of contraceptive implants to alleviate it. Contraception. 2008;78:73-8.

6. World Health Organization. High rates of unintended pregnancies linked to gaps in family planning services: New WHO study. 2019. https://www. who.int/news-room/detail/25-10-2019-high-rates-of-unintended-pregn ancies-linked-to-gaps-in-family-planning-services-new-who-study. Accessed 27 Apr 2020.

7. Summers C. Unintended pregnancy and abortion in Uganda. Guttmacher Inst. 2013

8. Cleland JG, Ndugwa RP, Zulu EM. Family planning in sub-Saharan Africa: progress or stagnation? Bull World Health Organ. 2011;89:137-43.

9. Tsui AO, Brown W, Li Q. Contraceptive Practice in sub-Saharan Africa. Popul Dev Rev. 2017;43:166-91.

10. Uganda Bureau of Statistics (UBOS) and ICF. Uganda demographic and health survey 2016. 2018. p 85-102. https://www.dhsprogram.com/ pubs/pdf/FR333/FR333.pdf. Accessed 17 Jan 2020.

11. Uganda Bureau of Statistics (UBOS) and ICF. Uganda demograpic and health survey 2011. ICF Int. 2012.p 1-461.

12. Guttmacher Institute. Contraception and unintended pregnancy in Uganda. 2017. https://www.guttmacher.org/sites/default/files/factsheet/ fb-contraception-and-unintended-pregnancy-in-uganda.pdf. Accessed on 27 Apr 2020.

13. World Health Organization. Family planning/contraception. Key fact sheet. 2018. https://www.who.int/news-room/fact-sheets/detail/famil y-planning-contraception. Accessed on 26 Apr2020.

14. Apanga PA, Adam MA. Factors influencing the uptake of family planning services in the Talensi District, Ghana. Pan Afr Med J. 2015;20:1-9.

15. Eliason S, Awoonor-Williams JK, Eliason C, Novignon J, Nonvignon J, Aikins M. Determinants of modern family planning use among women of reproductive age in the Nkwanta district of Ghana: a case-control study. Reprod Health. 2014;11:65.

16. Orach CG, Otim G, Aporomon JF, Amone R, Okello SA, Odongkara B, et al. Perceptions, attitude and use of family planning services in post conflict Gulu district, northern Uganda. Confl Health. 2015;9:24.

17. World Health Organization. Family planning/contraception. WHO [Internet]. World Health Organization [cited 18 Aug 2016]. http://www. who.int/mediacentre/factsheets/fs351/en.2018. https://www.who.int/ news-room/fact-sheets/detail/family-planning-contraception. Accessed on 25 Apr 2020.

18. Nalwadda G, Tumwesigye NM, Faxelid E, Byamugisha J, Mirembe F. Quality of care in contraceptive services provided to young people in two Ugandan districts: a simulated client study. PLOS ONE. 2011;6:e27908.

19. Peltzer K, Pengpid S. Contraceptive non-use and associated factors among university students in 22 countries. Afr Health Sci. 2015;15:1056-64.

20. Mehra D, Agardh A, Petterson KO, Östergren PO. Non-use of contraception: determinants among Ugandan university students. Glob Health Action. 2012:5:18599.

21. Ministry of Health Kampala Uganda. Uganda family planning costed implementation plan, 2015-2020. 2014. https://www.healthpolicypro ject.com/ns/docs/CIP_Uganda.pdf. Accessed 30 Oct 2020.
22. Okurut FN, Odwee JJAO, Adebua A. Determinants of regional poverty in Uganda. 2002.

23. UBOS, UNICEF, The World Bank. Poverty maps of Uganda. 2019. p 1-37.

24. UNFPA. Leaving no one behind in Karamoja. Issue Br 07. 2018. p 1-8.

25. Nakirijja DS, Xuili X, Kayiso MI. Socio-economic determinants of access to and utilization of contraception among rural women in Uganda: the case of Wakiso district. Health Sci J. 2018;12:1-20.

26. Ndayizigiye M, Fawzi MCS, Lively CT, Ware NC. Understanding low uptake of contraceptives in resource-limited settings: a mixed-methods study in rural Burundi. BMC Health Serv Res. 2017;17:209.

27. Frost JJ, Lindberg LD. Reasons for using contraception: perspectives of US women seeking care at specialized family planning clinics. Contraception. 2013;87:465-72.

28. Asiimwe JB, Ndugga P, Mushomi J, Menyenye Ntozi JP. Factors associated with modern contraceptive use among young and older women in Uganda; a comparative analysis. BMC Public Health. 2014;14:926.

29. Alano A, Hanson L. Women's perception about contraceptive use benefits towards empowerment: a phenomenological study in Southern Ethiopia. PLOS ONE. 2018;13:1-15.

30. Uganda Bureau of Statistics (UBOS) and ICF. Uganda demographic and health survey 2016. 2018.p 85-102.

31. Acock AC. A gentle introduction to Stata, Second Edition. 2008. http:// books.google.com/books?id=YZIQO0acuWwC\&pgis=1. Accessed 17 Jan 2020.

32. Hosmer DW, Lemesbow S. Goodness of fit tests for the multiple logistic regression model. Commun Stat Theory Methods. 1980;9:1043-69. https ://doi.org/10.1080/03610928008827941.

33. Solanke BL. Factors influencing contraceptive use and non-use among women of advanced reproductive age in Nigeria. J Health Popul Nutr. 2017;36:1-14. https://doi.org/10.1186/s41043-016-0077-6.

34. Moreira LR, Ewerling F, Barros AJD, Silveira MF. Reasons for nonuse of contraceptive methods by women with demand for contraception not satisfied: an assessment of low and middle-income countries using demographic and health surveys. Reprod Health. 2019;16:1-15.

35. Adhikari R, Acharya D, Ranabhat CL, Ranju KC. Factors associated with non-use of contraceptives among married women in Nepal. J Heal Promot. 2019;7:7-18.

36. Muhindo R, Okonya JN, Groves S, Chenault M. Predictors of contraceptive adherence among women seeking family planning services at reproductive health Uganda, Mityana Branch. Int J Popul Res. 2015;2015:1-8.

37. Andi JR, Wamala R, Ocaya B, Kabagenyi A. Modern contraceptive use among women in Uganda: an analysis of trend and patterns (1995-2011). Etude la Popul Africaine. 2014;28:1009-21.

38. Sable MR, Libbus MK, Chiu JE. Factors affecting contraceptive use in women seeking pregnancy tests: Missouri, 1997. Fam Plan Perspect. 2000;32:124-31.

39. Gakidou E, Vayena E. Use of modern contraception by the poor is falling behind. PLoS Med. 2007;4:0381-9.

40. Shakya HB, Dasgupta A, Ghule M, Battala M, Saggurti N, Donta B, et al. Spousal discordance on reports of contraceptive communication, contraceptive use, and ideal family size in rural India: a cross-sectional study. BMC Womens Health. 2018;18:1-14.

41. Utomo B, Alimoeso S, Park CB. Factors affecting the use and non use of contraception. Majalah Demografi Indones. 1983;10:ii.

42. Islam A. Prevalence and determinants of contraceptive use among employed and unemployed women in Bangladesh. Int J MCH AIDS 2016;5:92-102

43. Lwelamira J, Mnyamagola G, Msaki MM. Knowledge, Attitude and Practice (KAP) towards modern contraceptives among married women of reproductive age in Mpwapwa District, Central Tanzania. Curr Res J Soc Sci. 2012;4:235-45.

44. Mohammed A, Woldeyohannes D, Feleke A, Megabiaw B. Determinants of modern contraceptive utilization among married women of reproductive age group in North Shoa Zone, Amhara Region, Ethiopia. Reprod Health. 2014;11:13.

45. Kabagenyi A, Habaasa G, Rutaremwa G. Low contraceptive use among young females in Uganda: Does birth history and age at birth have an influence? Analysis of 2011 demographic and health survey. J Contracept Stud. 2016:1:1-12. 
46. Rankin K, Heard A, Diaz N, Box PO, Murray JL, World Health Organization (WHO), et al. Chapter 5. Data collector's guide. Lancet. 2016;59:1-99. https://doi.org/10.4236/ojpm.2013.32026.

47. Bankole A, Malarcher S. Removing barriers to adolescents' access to contraceptive information and services. Stud Fam Plan. 2010;41:117-24.

48. Osmani AK, Reyer JA, Osmani AR, Hamajima N. Factors influencing contraceptive use among women in Afghanistan: secondary analysis of Afghanistan Health Survey 2012. Nagoya J Med Sci. 2015;77:551-61.

49. Monteith RS, Anderson JE, Pineda MA, Santiso R, Oberle M. Contraceptive use and fertility in Guatemala. Stud Fam Plan. 1985;16:279-88.

50. Almalik M, Mosleh S, Almasarweh I. Are users of modern and traditional contraceptive methods in Jordan different? East Mediterr Health J. 2018;24:377-84

51. Aquino MP, Machado DL, Rodríguez J. A reader in Latina feminist theology: religion and justice. Austin: University of Texas Press; 2010.

52. Schenker JG. Women's reproductive health: monotheistic religious perspectives. Int J Gynecol Obstet. 2000;70:77-86.

53. Underwood C. Islamic precepts and family planning: the perceptions. Int Fam Plan Perspect. 2000;26:110-7.

54. Shabaik SA, Awaida JY, Xandre P, Nelson AL. Contraceptive beliefs and practices of American Muslim women. J Women's Health. 2019;28:976-83.

55. Raj A, Saggurti N, Balaiah D, Silverman JG. Prevalence of child marriage and its impact on the fertility and fertility control behaviors of young women in India. Lancet. 2009;373:1-20. https://doi.org/10.1016/S0140 $-6736(09) 60246-4$.

56. Mostafa Kamal SM. Decline in child marriage and changes in its effect on reproductive outcomes in Bangladesh. J Health Popul Nutr. 2012;30:317-30

57. Ewerling F, Lynch JW, Victora CG, van Eerdewijk A, Tyszler M, Barros AJD. The SWPER index for women's empowerment in Africa: development and validation of an index based on survey data. Lancet Glob Health. 2017:5:e916-23.

58. Nguyen N, Londeree J, Nguyen LH, Tran DH, Gallo MF. Reproductive autonomy and contraceptive use among women in Hanoi, Vietnam. Contracept X. 2019;1:100011.

59. Loll D, Fleming PJ, Manu A, Morhe E, Stephenson R, King EJ, et al. Reproductive autonomy and modern contraceptive use at last sex among young women in Ghana. Int Perspect Sex Reprod Health. 2019;45:1-12.
60. Viswan SP, Ravindran TKS, Kandala NB, Petzold MG, Fonn S. Sexual autonomy and contraceptive use among women in Nigeria: findings from the demographic and health survey data. Int J Women's Health. 2017:9:581-90

61. Magnusson BM, Masho SW, Lapane KL. Early age at first intercourse and subsequent gaps in contraceptive use. J Women's Health. 2012;21:73-9.

62. Tamang L, Raynes-Greenow C, McGeechan K, Black K. Factors associated with contraceptive use among sexually active Nepalese youths in the Kathmandu Valley. Contracept Reprod Med. 2017:2:1-8.

63. Olaolorun F, Seme A, Otupiri E, Ogunjuyigbe P, Tsui A. Women's fertility desires and contraceptive behavior in three peri-urban communities in sub Saharan Africa. Reprod Health. 2016;13:1-6. https://doi.org/10.1186/ s12978-016-0118-Z

64. Islam AZ. Factors affecting modern contraceptive use among fecund young women in Bangladesh: Does couples'joint participation in household decision making matter? Reprod Health. 2018;15:1-9.

65. Gupta N, Katende C, Bessinger R. Associations of mass media exposure with family planning attitudes and practices in Uganda. Stud Fam Plan. 2003;34:19-31.

66. Bajoga UA, Atagame KL, Okigbo CC. Media influence on sexual activity and contraceptive use: a cross sectional survey among young women in urban Nigeria. Afr J Reprod Health. 2015;19:100-10.

67. Bankole A, Rodriguez G, Westoff CF. Mass media messages and reproductive behaviour in Nigeria. J Biosoc Sci. 1996;28:227-39.

68. Rahman MD, Mondal MNI, Ali MK. A study on the factors affecting the use of contraception in Bangladesh. Int Res J Biochem Bioinform. 2011;7:178-83.

69. Hossain MK, Mondal MNI, Akter MN. Reproductive health rights of women in the rural areas of Meherpur District in Bangladesh. Med Reprod Infertil. 2011;12:23-32.

\section{Publisher's Note}

Springer Nature remains neutral with regard to jurisdictional claims in published maps and institutional affiliations.
Ready to submit your research? Choose BMC and benefit from:

- fast, convenient online submission

- thorough peer review by experienced researchers in your field

- rapid publication on acceptance

- support for research data, including large and complex data types

- gold Open Access which fosters wider collaboration and increased citations

- maximum visibility for your research: over $100 \mathrm{M}$ website views per year

At BMC, research is always in progress.

Learn more biomedcentral.com/submissions 